\title{
A simple solution for one of the cosmological constant problem by a topological field theory
}

\author{
Taisaku Mori* \\ Nagoya University \\ E-mail: mori.taisakuek.mbox.nagova-u.ac.jp

\section{Daisuke Nitta} \\ Nagoya University \\ E-mail: nitta.daisukedg.mbox.nagova-u.ac.ip
}

\section{Shin'ichi Nojiri}

Nagoya University, Kobayashi-Maskawa Institute for the Origin of Particles and the Universe E-mail: nojiriephys.nagova-u.ac.jp

\begin{abstract}
We propose a simple and totally covariant model which may solve the fine-tuning problem. The model is given by a topological field theory and the model has an infinite numbers of the BRS symmetries. The BRS symmetries are, however, spontaneously broken in general. In this proceeding, we investigate the BRS symmetry in more details and show that there is one and only one BRS symmetry which is not broken and the unitarity can be guaranteed. In the model, the quantum problem of the vacuum energy, which may be identified with the cosmological constant, reduces to the classical problem of the initial condition. We investigate the cosmology given by the model and specify the region of the initial conditions which could be consistent with the evolution of the universe. We also show that there is a stable solution describing the de Sitter space-time, which may explain the accelerating expansion in the current universe.
\end{abstract}

The 3rd International Symposium on a Quest for the Origin of Particles and the Universe" 5-7 January 2017

Nagoya University, Japan

\footnotetext{
* Speaker.
} 


\section{Introduction}

The energy density generating the accelerating expansion of the universe is called as dark energy. The $\Lambda$ CDM model of the dark energy could be a cosmological term with a small cosmological constant, $\Lambda^{1 / 4} \sim 10^{-3} \mathrm{eV}$. The cosmological term can be regarded as the energy density of the vacuum in quantum field theory $\rho_{\text {vacuum }}$. When we introduce the cutoff scale $\Lambda_{\text {cutoff }}$, which might be the Planck scale, the vacuum energy $\sim \Lambda_{\text {cutoff }}^{4}$ is much larger than the observed value $\left(10^{-3} \mathrm{eV}\right)^{4}$ of the energy density in the universe. We may use the counter term in order to obtain the observed very small vacuum energy $\left(10^{-3} \mathrm{eV}\right)^{4}$ but very very fine-tuning is necessary and it looks extremely unnatural.

\section{Simple model}

In [W], motivated by the unimodular gravity theories, a new model has been proposed. One of the action of this model is given by,

$$
S^{\prime}=\int d^{4} x \sqrt{-g}\left\{\frac{R}{2 \kappa^{2}}-\Lambda-\lambda+\partial_{\mu} \lambda \partial^{\mu} \varphi-\partial_{\mu} b \partial^{\mu} c\right\}+S_{\text {matter }}
$$

Here $\lambda$ and $\varphi$ are scalar fields, and $b$ and $c$ are also scalar fields but they are fermionic (Grassmann odd) and later $b$ is identified with the anti-ghost and $\mathrm{c}$ with ghost and $R$ is the Ricci scalar and $\kappa$ is gravitational coupling constant. In (R.D), we express the action of matters by $S_{\text {matter }}$ can be that of arbitrary model. When we redefine the scalar field $\lambda$ by $\lambda \rightarrow \lambda-\Lambda$, the action (ㅍ.J) is rewritten as,

$$
S^{\prime}=\int d^{4} x \sqrt{-g}\left\{\frac{R}{2 \kappa^{2}}-\lambda+\partial_{\mu} \lambda \partial^{\mu} \varphi-\partial_{\mu} b \partial^{\mu} c\right\}+S_{\text {matter }} \equiv \int d^{4} x \sqrt{-g}\left\{\mathscr{L}+S_{\text {matter }}\right\} .
$$

Then the obtained action (2.2) does not include the constant $\Lambda$, which tells that the constant $\Lambda$ does not affect the dynamics. The model in (․ㅔ) includes ghosts [四], which generates the negative norm states in the quantum theory and therefore the model is inconsistent but the negative norm states can be excluded by defining the physical states by using the BRS symmetry [B]]. In fact, the action is invariant under the infinite numbers of the BRS transformation,

$$
\delta \lambda=\delta c=0, \quad \delta \varphi=\varepsilon c, \quad \delta b=\varepsilon\left(\lambda-\lambda_{0}\right) .
$$

Here $\varepsilon$ is a fermionic parameter and $\lambda_{0}$ is a solution of the equation,

$$
0=\nabla^{\mu} \partial_{\mu} \lambda
$$

which can be obtained by the variation of the action (2. $($ ) with respect to $\varphi$. We now impose the following gauge condition in order to fix the gauge symmetry,

$$
1+\nabla_{\mu} \partial^{\mu} \varphi=0
$$

Then the gauge-fixing Lagrangian [ [ [ ] is given by the BRS transformation ([2.3]) of $-b\left(1+\nabla_{\mu} \partial^{\mu} \varphi\right)$. In fact, we find

$$
\delta\left(-b\left(1+\nabla_{\mu} \partial^{\mu} \varphi\right)\right)=\varepsilon\left(\mathscr{L}+\lambda_{0}+(\text { total derivative terms })\right) .
$$


Therefore the Lagrangian density (I.2) is surely BRS exact up to the total derivative terms if $\lambda_{0}=0$ and we find that the theory in ([2.2) is surely a topological field theory. Then by using Eq. ([2.3)), $\lambda-\lambda_{0}$ is BRS exact, which tells that the vacuum expectation value of $\lambda-\lambda_{0}$ must vanish [ [8, $Q$, [0] If the vacuum expectation value of $\lambda-\lambda_{0}$ does not vanish, the BRS symmetry is spontaneously broken. We should note that there is only one unbroken BRS symmetry in when $\lambda=\lambda_{0}$. Therefore in the real world, only one $\lambda_{0}$ is chosen and the corresponding BRS symmetry is not broken.

\section{Cosmological evolution}

The values $\lambda$ or $\Lambda+\lambda$ could be determined by the initial conditions in the classical theory. Then in the following, we investigate the cosmology given by the model (2. $(2)$ and specify the region of the initial conditions which could be consistent with the evolution of the observed universe. We may assume the FRW metric with flat spacial part,

$$
d s^{2}=-d t^{2}+a(t)^{2} \sum_{i=1}^{3}\left(d x^{i}\right)^{2}
$$

and $\lambda$ and $\varphi$ are assumed to only depend on the time coordinate $t$. In (B.]), $a(t)$ is called as the scale factor. By the variation of $\lambda$ in the action (‥J), we obtain Eq. (‥5). Neglecting the contributions from matters, we consider the FRW cosmology. Then the first and second FRW equations are

$$
\frac{3}{\kappa^{2}} H^{2}=\Lambda+\lambda-\frac{d \lambda}{d t} \frac{d \varphi}{d t},-\frac{1}{\kappa^{2}}\left(3 H^{2}+2 \frac{d H}{d t}\right)=-\Lambda-\lambda-\frac{d \lambda}{d t} \frac{d \varphi}{d t} .
$$

We find that there is a solution, where $\lambda$ is a constant $\lambda=\lambda_{1}$. In fact, $\lambda=\lambda_{1}$ is one of the solution of ([2.4) with FRW metric (B.2). Then Eq. (3.2) tells that $H$ is a constant, $H=H_{0}$ and therefore the space-time is the de-Sitter space-time. We now investigate the stability of the solution under the de Sitter space-time. For this purpose, we consider the perturbation from the solution,

$$
H=H_{0}+\delta H, \quad \lambda=\lambda_{1}+\delta \lambda, \quad \varphi=\varphi_{\text {de Sitter }}+\delta \varphi .
$$

Then by using ([2.4), ([2.5), and (B.2), we can write the equations in the matrix form,

$$
\left(\begin{array}{c}
\delta \dot{\lambda} \\
\delta \dot{\eta} \\
\delta \ddot{\varphi}
\end{array}\right)=A\left(\begin{array}{c}
\delta \lambda \\
\delta \eta \\
\delta \dot{\varphi}
\end{array}\right), \quad A \equiv\left(\begin{array}{ccc}
0 & 1 & 0 \\
0 & -3 H_{0} & 0 \\
\frac{\kappa^{2}}{18 H_{0}^{2}} & -\frac{\kappa^{2}}{54 H_{0}^{3}} & -3 H_{0}
\end{array}\right), \eta \equiv \dot{\lambda} .
$$

The eigenvalues of the matrix $A$ is given by $-3 H_{0}$ and two 0 's. Because there is not positive eigenvalues, the solution is stable or at least quasi-stable. We now investigate what could be the initial condition corresponding to the value of the vacuum energy in the present universe. In the radiation-dominated era, the matter-dominated era, and the dark energy-dominated era, the scale factor is given by

$$
a(t)=a_{\mathrm{rad}} t^{1 / 2}, a(t)=a_{\mathrm{mat}} t^{2 / 3}, a(t)=a_{\Lambda} \mathrm{e}^{H_{0} \sqrt{\Omega_{\Lambda}} t} .
$$

respectively. Here $a_{\mathrm{rad}}, a_{\mathrm{mat}}$, and $a_{\Lambda}$ are constants depending on the energy density of the radiation, the matter density, and the dark energy density, respectively, and $H_{0}$ is Hubble rate in the current 
universe and $\Omega_{\Lambda}$ is the dark energy density parameter. In the FRW universe, from (2.4 (B.5), the scalar fields $\lambda(t)$ and $\varphi(t)$ in the radiation-dominated era are given by

$$
\varphi(t)=\varphi_{\mathrm{rad}}(t) \equiv \varphi_{\mathrm{rad} 2}-\frac{2 \varphi_{\mathrm{rad} 1}}{a_{\mathrm{rad}}^{3}} t^{-1 / 2}+\frac{1}{5} t^{2}, \quad \lambda(t)=\lambda_{\mathrm{rad}}(t) \equiv \lambda_{\mathrm{rad} 1}-\frac{2 \lambda_{\mathrm{rad} 2}}{a_{\mathrm{rad}}^{3}} t^{-1 / 2} .
$$

Here, $\varphi_{\mathrm{rad} 1}, \varphi_{\mathrm{rad} 2}, \lambda_{\mathrm{rad} 1}, \lambda_{\mathrm{rad} 2}$ are some constant. In the matter dominant era and the dark energy dominant era, we can also express $\varphi_{\text {mat }}(t), \varphi_{\Lambda}(t), \lambda_{\text {mat }}(t)$ and $\lambda_{\Lambda}(t)$ by using $\varphi_{\text {rad1 }}, \varphi_{\text {rad2 }}, \lambda_{\text {rad1 } 1}, \lambda_{\text {rad2 }}$ if we assume the following approximations. Where the radiation-dominated era transited to the matter-dominated era at the time $t=t_{1}$, we assume $\varphi_{\text {mat }}\left(t_{1}\right)=\varphi_{\text {rad }}\left(t_{1}\right)$ and $\dot{\varphi}_{\text {mat }}\left(t_{1}\right)=\dot{\varphi}_{\text {mat }}\left(t_{1}\right)$. Similarly, we connect the matter dominant era to the dark energy dominant era and express all era of scalar field only by using $\varphi_{\mathrm{rad} 1}, \varphi_{\mathrm{rad} 2}, \lambda_{\mathrm{rad} 1}, \lambda_{\mathrm{rad} 2}$. We impose the two constraints on the above constants which may explain the initial condition of the scalar fields. First constraint could be obtained by requiring $\Lambda+\lambda$ should become a constant corresponding to the cosmological constant. Second constraint requires that the matter should be surely dominant compared with the contributions from $\lambda$ and $\varphi$. Then we may have the following constraint[ [W],

$$
\begin{gathered}
\Lambda+\lambda_{\text {rad } 1}-\lambda_{\text {rad2 }} \times\left(3.1 \times 10^{65}\left[\mathrm{eV}^{-1}\right]\right) \sim 10^{-11}\left[\mathrm{eV}^{4}\right], \quad\left|\lambda_{\text {rad2 }}\right| \ll 10^{-47}\left[\mathrm{eV}^{5}\right], \\
\left|\lambda_{\text {rad } 2}\left(\varphi_{\text {rad } 1}+1.4 \times 10^{16}\left[\mathrm{eV}^{-1}\right]\right)\right| \ll 10^{-23}\left[\mathrm{eV}^{4}\right], \quad\left|\lambda_{\text {rad } 2} \varphi_{\text {rad } 1}\right| \ll 10^{-62}\left[\mathrm{eV}^{4}\right] .
\end{gathered}
$$

The first constraint in (B.7) seems to tell that we need the fine tuning for the initial conditions.

\section{Acknowledgments.}

The authors are indebted S. Akagi, K. Ichiki, T, Katsuragawa, and N. Sugiyama. This work is supported (in part) by MEXT KAKENHI Grant-in-Aid for Scientific Research on Innovative Areas "Cosmic Acceleration" (No. 15H05890) (D.N and S.N.).

\section{References}

[1] S. Nojiri, Mod. Phys. Lett. A 31 (2016) no.37, 1650213 doi:10.1142/S0217732316502138 [arXiv:1601.02203 [hep-th]].

[2] B. Shlaer, arXiv:1411.8006 [gr-qc].

[3] C. Becchi, A. Rouet and R. Stora, Annals Phys. 98 (1976) 287. doi:10.1016/0003-4916(76)90156-1

[4] T. Kugo and I. Ojima, Phys. Lett. B 73 (1978) 459. doi:10.1016/0370-2693(78)90765-7

[5] T. Kugo and I. Ojima, Prog. Theor. Phys. Suppl. 66 (1979) 1. doi:10.1143/PTPS.66.1

[6] E. Witten, Commun. Math. Phys. 117 (1988) 353. doi:10.1007/BF01223371

[7] T. Kugo and S. Uehara, Nucl. Phys. B 197 (1982) 378. doi:10.1016/0550-3213(82)90449-7

[8] N. Nakanishi, Prog. Theor. Phys. 35 (1966) 1111. doi:10.1143/PTP.35.1111

[9] N. Nakanishi, Prog. Theor. Phys. 49 (1973) 640. doi:10.1143/PTP.49.640

[10] B. Lautrup, Kong. Dan. Vid. Sel. Mat. Fys. Med. 35 (1967) 11, .

[11] P. A. R. Ade et al. [Planck Collaboration], Astron. Astrophys. 594 (2016) A13 doi:10.1051/0004-6361/201525830 [arXiv:1502.01589 [astro-ph.CO]]. 